\title{
La gestión pública orientada a la inversión y al crecimiento
}

\author{
Ricardo Martner \\ Director, Dirección de \\ Gestión Pública y Regulación, \\ Instituto Latinoamericano \\ $y$ del Caribe \\ de Planificación Económica \\ y Social (ILPES) \\ rmartner@eclac.cl
}

En este artículo se pone de relieve el papel central que desempeñan mercados imperfectos o incompletos en la propagación y persistencia de las situaciones recesivas. La persistencia generada por la volatilidad de la demanda es un hecho cierto que sólo puede ser atenuado por políticas sostenibles de estímulo al nivel de actividad. Las reglas macrofiscales, importantes para mejorar la mermada credibilidad de la acción pública, deben combinar dos principios fundamentales: la responsabilidad y la estabilidad. Esto supone preservar los mecanismos de regulación para estabilizar fluctuaciones macroeconómicas excesivas. Lo mejor que pueden hacer las autoridades es prevenir tales fluctuaciones con políticas flexibles de intervención. El nuevo paradigma de gestión pública por resultados supone así establecer reglas fiscales claras, con metas de mediano plazo y capacidad de estabilización en el corto plazo, pero también avanzar en la asignación de proporciones crecientes del gasto público de manera plurianual. Para garantizar un crecimiento económico estable y una adecuada ejecución de los planes y programas votados por la ciudadanía, la gestión pública enfrenta tres desafíos fundamentales: respetar una regla macrofiscal a lo largo del ciclo, identificar la aparición de déficit estructurales y eliminar el tradicional sesgo contra la inversión. En este artículo se reseñan algunas experiencias recientes en que se ha procurado enfrentar explícitamente estos serios obstáculos, con normativas legales que toman en cuenta las consecuencias de las incertidumbres asociadas a la marcha cíclica de la economía y a la estimación de su potencial de crecimiento de largo plazo. 


\section{I}

\section{Introducción}

La filosofía económica dominante postula el carácter esencialmente transitorio de las perturbaciones macroeconómicas y el carácter perverso de las intervenciones públicas. Las políticas de estabilización serían totalmente ineficaces en su objetivo de aumentar el crecimiento de largo plazo, y, más aun, agudizarían las fluctuaciones. Sería preferible entonces no entorpecer el normal ajuste hacia el equilibrio: hacer y deshacer podría ser más costoso que no hacer nada. Según esta ley de abstinencia, las buenas políticas son aquellas que otorgan credibilidad al aislarse de las presiones intervencionistas. Congruentes con esta filosofía, en América Latina la mayoría de las reformas del sector público y las recientes leyes de responsabilidad fiscal han procurado eliminar o restringir el papel de regulación macroeconómica que se les atribuye tradicionalmente a las finanzas públicas.

Llama la atención, sin embargo, la gran distancia que separa la certidumbre de las recomendaciones con la intensidad de la discusión teórica y la fragilidad del análisis empírico. Por una parte, la teoría económica tiene debates fundamentales sobre las causalidades en la explicación de las patologías macroeconómicas, como la inflación, el desempleo y la propia insolvencia fiscal. Por otra, el problema de la identificación es lo suficientemente grave como para incitar a los economistas a la modestia; la complejidad de la interacción entre variables observables y no observables hace difícil identificar regularidades estadísticas y, por lo tanto, demostrar empíricamente cualquier hipótesis inicial. Según Greenspan (1996), no existe un modelo simple de la economía que pueda efectivamente explicar los niveles de producto interno bruto (PIB), de empleo y de inflación. En principio, podría haber un conjunto increíblemente complejo de ecuaciones que lo haga. Pero no hemos sido capaces de encontrarlas, y no creemos tampoco que alguien lo haya hecho.

La coexistencia de situaciones recesivas prolongadas, de entidades monetarias con algún grado de autonomía en el establecimiento de sus metas y de leyes de responsabilidad fiscal extremadamente rígidas ha producido situaciones curiosas, en que a la autoridad monetaria sólo le preocupa la evolución de la inflación y al sector público las metas de corto plazo de saldo, por cierto imposibles de cumplir en un entorno incier- to. La ausencia de coordinación de metas e instrumentos puede llevar así a una cierta dilución de responsabilidades, puesto que ninguna autoridad tiene objetivos ni debe rendir cuentas por lo que ocurre en el ámbito del nivel de actividad, la estabilidad real y el desempleo. En el último tiempo, la combinación de políticas aplicadas en la región no ha sido siempre la más adecuada, con consecuencias negativas y considerables para el desempeño agregado. La política fiscal también tiene un papel no menor en la prevención de fluctuaciones excesivas, y este aspecto no se ha considerado en las reformas recientes.

Las dificultades para conocer la estructura de la economía y para prever las consecuencias de los cambios no tienen por qué incitar a una gestión macroeconómica que se limite a administrar. La incertidumbre obliga a las autoridades a enfrentar, de manera recurrente, dos problemas de compleja solución: por una parte, contener los estados de ánimo de los agentes macroeconómicos (como la euforia o el pesimismo), ofreciendo una garantía de estabilidad que prolongue el horizonte temporal de las decisiones y, por otra parte, el conservadurismo sistemático y la pérdida de oportunidades que éste conlleva.

El presente artículo, que sintetiza un trabajo más detallado, consta de tres secciones, además de esta introducción. En la sección II se revisa la literatura reciente que hace hincapié en el papel que desempeñan mercados imperfectos o incompletos en la propagación y persistencia de las situaciones recesivas. La volatilidad de la demanda induce a una subutilización de los factores de producción, lo que a su vez genera persistencia e histéresis. Para muchos autores, la única manera eficaz de atenuar la volatilidad y la incertidumbre y de enfrentar la debilidad persistente de la demanda global y sus devastadores efectos sobre la oferta agregada, es a través de políticas sostenibles de estímulo fiscal.

En la sección III se plantea que las reglas macrofiscales deben respetar dos principios fundamentales, como son la responsabilidad y la estabilidad. El primero ha sido, por lo general, el único objetivo de las reformas recientes de nuestros países; el segundo ha tenido un espacio bastante menor. Este último, entendido como el objetivo de mantener a un nivel alto la 
producción y el empleo, no es incorporado explícitamente en las leyes de responsabilidad fiscal de América Latina. Sin embargo, la credibilidad de las políticas se garantiza combinando adecuadamente tales principios, lo que supone disponer de mecanismos de intervención para estabilizar fluctuaciones macroeconómicas excesivas. La aplicación de reglas anticíclicas en tiempos normales, pero preservando la discrecionalidad para enfrentar situaciones imprevistas, puede representar una respuesta a los desafíos planteados por la extraordinaria volatilidad de las economías latinoamericanas.

La sección IV retoma algunos temas relativos al nuevo modelo de gestión pública y al nexo entre pla- nificación y presupuesto, entendido como la necesidad de asignar proporciones crecientes del gasto público de manera plurianual para cumplir eficientemente los planes y programas públicos. La gestión pública moderna debe enfrentar tres desafíos: respetar una regla macrofiscal a lo largo del ciclo, identificar con anticipación la aparición de déficit estructurales para no realizar ajustes abruptos, y eliminar el tradicional sesgo contra la inversión que se produce cuando existen contracciones de gasto. La tarea es difícil, pero no imposible: reseñamos aquí algunas experiencias recientes en que se ha procurado enfrentar explícitamente estos graves obstáculos al nuevo paradigma del modelo de gestión por resultados.

\section{II}

\section{Fluctuaciones macroeconómicas}

\section{y bienestar agregado}

\begin{abstract}
"La Economía es la ciencia de pensar en términos de modelos, unida al arte de escoger los modelos que son idóneos para el mundo contemporáneo".
\end{abstract}

John Maynard Keynes, Carta a Roy Harrod, 1938
Para algunos, la fuente principal de las fluctuaciones macroeconómicas es el carácter distorsionador de las intervenciones públicas. Como lo plantea la célebre máxima de Friedman (1968), 'las políticas deben precaverse en sí mismas de ser una fuente importante de perturbaciones económicas'. ${ }^{1}$ El mercado se autorregula, y las fluctuaciones son necesarias para asegurar la eficiencia global de la economía. Para otros, en cambio, tales fluctuaciones son inherentes a fallas de mercado y dañinas para el crecimiento; las políticas públicas deben ser muy activas para aminorarlas cuando existen mercados incompletos. A la hora de escoger el modelo apropiado para nuestro mundo contemporáneo, corresponde entonces explicar estas dos visiones antagónicas.

Los economistas emblemáticos de las escuelas de los nuevos clásicos basan sus teorías en modelos simples de mercados caracterizados por información y

\footnotetext{
${ }^{1}$ En este artículo, las citas entre comillas simples corresponden a traducciones del idioma original hechas por el autor.
}

competencia perfecta, con ausencia de costos de transacción y la presencia de un conjunto completo de mercados. Existe un agente representativo, lo que entre otras cosas elimina los problemas de riesgo y de información asimétrica. Para explicar las fluctuaciones macroeconómicas, los teóricos de la corriente de los nuevos clásicos ponen de relieve las perturbaciones tecnológicas, las sustituciones intertemporales entre trabajo y ocio, o los ciclos reales provenientes de cambios en la oferta agregada. Los clásicos continúan interpretando el ciclo económico dentro de un modelo con mercados sin fricciones a la Arrow-Debreu. En una economía walrasiana pura, el nivel de producto que prevalece con precios totalmente flexibles es óptimo.

La incapacidad de los herederos de Walras de describir el mundo real fue denunciada por el mismo Keynes (1936) en su Teoría General: 'nuestra crítica de la economía clásica consiste menos en revelar los errores lógicos en su análisis que en poner en evidencia el hecho de que las hipótesis implícitas en él nunca se realizan, y por ello es incapaz de resolver los problemas del mundo concreto'. Las imperfecciones son 
la principal diferencia entre el mundo real y el modelo walrasiano de Arrow-Debreu. En palabras de Greenwald y Stiglitz (1993), 'dejar estos aspectos fuera del modelo es como dejar al príncipe Hamlet fuera de la obra'.

Durante algunos períodos, a menudo largos, existe un exceso de oferta de trabajo al nivel de salario real prevaleciente. En otras palabras, existe desempleo involuntario. El nivel agregado de la actividad económica fluctúa marcadamente, ya sea que ésta se mida por el grado de uso de la capacidad, por el PIB o por la tasa de desempleo; estas fluctuaciones son de mayor magnitud que las que pudieran provenir de cambios de corto plazo en la tecnología, en los gustos de los consumidores o en la demografía, por ejemplo.

Como lo recuerda Tobin (1993), el gran debate entre Keynes y sus oponentes se refería a la eficacia de los mecanismos naturales de ajuste de las economías de mercado para restaurar el equilibrio de pleno empleo, cuando alguna perturbación negativa de demanda ha sacado a la economía de ese equilibrio. Keynes y los keynesianos afirmaban que esos mecanismos eran débiles, posiblemente inexistentes o perversos y que, por lo tanto, se necesitaba la intervención de políticas públicas. Para Blanchard (1996), en medio de la Gran Depresión era irresponsable esperar que la economía volviera por sí misma a su nivel natural, y tratar de equilibrar el presupuesto público no sólo era estúpido, sino también peligroso. En este marco, la premisa es que el desempleo persistente y las fluctuaciones económicas son problemas centrales y continuos: las recesiones y depresiones representan fallas de mercado a gran escala, según la expresión de Mankiw (1993).

Muchos autores hacen hincapié en el papel que pueden desempeñar mercados imperfectos o incompletos en la generación, amplificación, propagación y persistencia de las fluctuaciones macroeconómicas (Greenwald y Stiglitz, 1989 y 1993; Stiglitz, 1999; Dreze, 1997 y 2000). Un tema común es que en mercados que carecen de mecanismos de regulación automática, las cantidades varían mucho porque los precios cambian muy poco y muy tarde. Estas imperfecciones en los ajustes del mercado están en el centro de las explicaciones de los procesos recesivos prolongados. Diversos elementos pueden explicar la ausencia de mercados que se autoequilibren: situaciones de monopolio, incertidumbre, costos de transacción, costos hundidos y costos de información, entre otros. Nos concentraremos en seguida en las dos primeras explicaciones.
Las fluctuaciones macroeconómicas pueden ser las consecuencias perversas de condiciones no competitivas (Mankiw; 1985, 1989 y 1993; Blanchard y Kiyotaki, 1987; Romer, 1993). En un régimen de competencia perfecta, las empresas bajan sus precios en respuesta a una reducción de la demanda agregada, evitando la caída de la producción. Pero es posible que pequeñas barreras en los procesos de ajuste de precios y salarios basten para transformar reducciones de poca magnitud de la demanda agregada en costosas recesiones. Las fricciones mencionadas hacen que, para cada empresa individual, las ganancias de bajar sus propios precios sean pequeñas. En un régimen de competencia monopolística ${ }^{2}$ las empresas fijan sus propios precios y aceptan las ventas reales como una restricción, en contraste con un régimen de competencia perfecta, en el que empresas competitivas tomadoras de precios pueden decidir sobre su producción.

En un régimen de competencia monopolística la caída de la demanda se traduce en precios que no cambian y en una recesión. Las empresas no tienen muchos incentivos para reducir sus precios cuando la demanda por sus bienes es menor, por lo que sus decisiones tienen externalidades. Como plantean Blanchard y Kiyotaki (1987), la externalidad de demanda agregada indica que las decisiones de las empresas individuales afectan a todas las otras a través de la demanda agregada. ${ }^{3}$ Podemos definir la externalidad de demanda agregada como la ganancia de bienestar que se daría si hubiese un cambio desde un régimen de equilibrio monopolístico a otro de competencia perfecta, puesto que en este último estado la demanda agregada sería más alta.

Cuando se combina un régimen de competencia monopolística con rigideces de precios, pequeños costos (de segundo orden) en los ajustes de precios pue-

\footnotetext{
${ }^{2}$ El concepto de competencia monopolística, contradictorio en sus términos, se refiere a una economía compuesta por firmas que producen bienes que son sustitutos imperfectos de los demás bienes (Blanchard y Kiyotaki, 1987). Como resultado, cada empresa tiene algún poder monopólico y, por ende, la facultad de fijar sus precios. La condición es que cada firma tiene una función objetivo diferenciable en sus propios precios, es decir, que puede cambiarlos marginalmente con respecto a sus competidores sin que sus ventas caigan a cero.

${ }^{3}$ En palabras de esos autores: 'si el punto de partida es un equilibrio de competencia monopolística y una firma reduce su precio, ello llevaría a una pequeña baja del nivel de precios y a un pequeño aumento de la demanda agregada. Como las otras empresas y los consumidores se beneficiarían de este incremento de la demanda, la firma que redujo su precio no puede capturar todos los beneficios de su acción y por lo tanto no tiene incentivos para hacerlo'.
} 
den provocar grandes cambios (de primer orden) en el producto, en caso de que se generen variaciones nominales. Debido a las distorsiones preexistentes propias de la fijación monopólica de precios, los beneficios para la sociedad ocasionados por una caída de los precios pueden ser importantes, aunque sean pequeños para la empresa. La microeconomía de los ajustes de precios es decisiva para la macroeconomía de las rigideces nominales.

La explicación de las fluctuaciones se encontraría en el comportamiento averso al riesgo de las empresas (Greenwald y Stiglitz, 1993). Las decisiones de éstas se ven afectadas por su percepción de los riesgos, asociados con la incertidumbre sobre las consecuencias de sus acciones y sobre el valor de sus activos. Al menos tres factores influyen en la evaluación de los riesgos por parte de las empresas.

El primero es la situación de la economía global. Cuando existe pesimismo ante la situación prevista, esta propia percepción tiene consecuencias reales, puesto que afecta a todas las decisiones de la empresa, desde la fijación de precios hasta el gasto en inversión, pasando por el volumen de empleo. En otras palabras, las expectativas influyen fuertemente en las decisiones y generan efectos multiplicadores que pueden alcanzar una gran magnitud. En palabras de Keynes (1936), 'la sabiduría universal enseña que es mejor para la propia reputación fracasar con las convenciones que tener éxito en contra de ellas'. La formación de expectativas es un fenómeno eminentemente social, y su autovalidación es uno de los riesgos mayores del sistema capitalista moderno.

La incertidumbre significa que el entorno económico del futuro no se conoce hoy. Los mercados completos representan una utopía según la cual los agentes económicos pueden intercambiar todos los bienes y servicios de manera condicionada al entorno económico futuro. Los mercados son incompletos cuando la cobertura de riesgos es limitada, es decir en el mundo real. Así, una relación macroeconómica fundamental, el equilibrio entre el ahorro y la inversión, se ve constantemente perturbada por el grado de incertidumbre percibido por los agentes económicos, puesto que de ello va a depender la realización efectiva del gasto en inversión.

Un segundo factor tiene que ver con la posición de los activos líquidos de las empresas. En un mundo en que existe racionamiento de crédito y comportamiento averso al riesgo la liquidez de la empresa adquiere gran importancia. La posición de liquidez es afectada por las utilidades, que representan un residuo de decisiones anteriores en materia de precios y cantidades. Para mantener el mismo nivel de costos en un contexto recesivo, con menor liquidez debido a utilidades inferiores, las empresas deben endeudarse más. Pero esta mayor deuda ocasiona una probabilidad más alta de que los futuros ingresos no sean suficientes para enfrentar las nuevas obligaciones. En consecuencia, percibir utilidades menores en un contexto recesivo y con racionamiento de crédito induce a las empresas a invertir menos y a producir menos.

Un tercer elemento importante es el cambio en los precios relativos, que tiene efectos importantes en el acceso al endeudamiento, en las tasas de interés pagadas y, por lo tanto, en la liquidez y la riqueza neta de las empresas. Un aumento de las tasas de interés erosiona muy rápidamente la riqueza neta de empresas ya endeudadas. La alta velocidad con que se ajustan el precio de los activos y las tasas de interés a las perturbaciones, combinada con las imperfecciones en el mercado de capitales que limitan la capacidad de las empresas para diversificar los riesgos, tiene profundas implicaciones para la oferta agregada de la economía.

Como los diversos precios se determinan de distintas maneras, y se ajustan a distintas velocidades, las perturbaciones conducen a cambios de gran magnitud en los precios relativos, que a su vez exacerban fuertemente las fluctuaciones macroeconómicas. La curva de oferta agregada se desplaza marcadamente hacia abajo si la economía entra en una recesión. El riesgo de producir aumenta y la capacidad de las empresas para enfrentar este riesgo merma.

Dada una perturbación contractiva de la demanda agregada, una eventual deflación de salarios y precios no hace que la economía retorne a su nivel de pleno empleo, ni siquiera en un régimen de competencia perfecta. Para Stiglitz (1999), la simultaneidad de grandes caídas de los precios y de los salarios reales y del nivel de actividad en algunos países durante la reciente crisis asiática ha vuelto a demostrar que la flexibilidad de precios y salarios en presencia de imperfecciones de mercado puede tener efectos negativos mucho mayores que los analizados en la literatura tradicional.

Para Dreze (2000), 'la incertidumbre y los mercados incompletos generan una volatilidad de la demanda y una rigidez de precios y salarios cuya conjunción conduce a equilibrios de oferta múltiples y restringidos, que reflejan una insuficiencia de coordinación que puede generar persistencia'. La disponibilidad de los factores de producción fija un límite 
superior a la producción, pero no existe límite inferior. Hay múltiples y prolongados períodos de subutilización o despilfarro de recursos tan importantes como el trabajo y la capacidad de producción.

Existe una perfecta sincronía entre la producción y la inversión, lo que implica que la volatilidad de la segunda repercute en la variabilidad de la primera. Para la empresa, el hecho de posponer la decisión de invertir tiene consecuencias de segundo orden sobre los beneficios esperados, pero consecuencias de primer orden si se reduce significativamente la demanda global de inversión. La mera existencia de mercados incompletos, entonces, es razón suficiente para generar volatilidad en la demanda agregada. La volatilidad genera subutilización de los recursos existentes y, al postergar la inversión, de los recursos futuros.

Si hay subutilización, ésta persistirá por ausencia de coordinación mientras no cambien las condiciones del mercado. La subutilización genera persistencia, por al menos tres razones: un débil nivel de actividad hoy tiende a alimentar expectativas de un débil nivel de actividad mañana; un bajo nivel de inversión hoy reduce el potencial de oferta de mañana; y un débil nivel de actividad hoy tiende a empeorar la situación financiera de mañana, pues las rigideces de los precios pueden impedir la recuperación de los márgenes de ganancia.

Es importante diferenciar el concepto de fallas de mercado que se asocia a la existencia de mercados imperfectos (y por lo tanto perfectibles con la introducción de competencia o de una regulación adecuada) y la noción de mercados incompletos, la que se explica por la existencia de expectativas probabilísticas en un mundo con cobertura limitada de riesgos. A su vez, estas expectativas probabilísticas, perfectamente racionales, se asocian a la incertidumbre respecto a los escenarios futuros. Ya no se trata de anticipar situaciones a partir de un modelo macroeconómico que todo el mundo conoce, como en la teoría neoclásica con expectativas racionales, sino de asignar probabilidades en un contexto de incertidumbre.

El programa, entonces, no se limita a promover la competencia, sino también a reducir la volatilidad. De lo expuesto hasta aquí se desprenden tres conclusiones de política (Dreze, 2000):

i) Es necesario buscar soluciones a la volatilidad de la demanda. Se debe estar consciente en todo momento de la posibilidad de que se subutilicen los recursos de trabajo y de capacidad de producción. La vía natural para garantizar que las políticas de estímulo de la demanda sean sostenibles consiste en concentrarlas en inversiones que tengan rendimientos sociales adecuados, como inversiones en vivienda social, renovación urbana, transporte urbano. El hecho de promover estas inversiones en períodos en que otras inversiones privadas se han reducido transitoriamente es una manera eficaz de atenuar la volatilidad, la debilidad persistente de la demanda global y sus devastadores efectos sobre la oferta agregada.

ii) La existencia de rigideces salariales y de precios es inevitable, lo que hace que las políticas de ajuste automático sean muy costosas. Es preciso atenuar los efectos de las rigideces con políticas específicas y transitorias, como mecanismos de moderación salarial ex ante para enfrentar situaciones recesivas, impuestos sobre el trabajo variables según la tasa de desempleo y políticas destinadas a aliviar la situación financiera de las PYME.

iii) No se puede desestimar los problemas de coordinación y de asimetría de mercados. La posibilidad de una insuficiencia de coordinación está siempre presente. El vicio de los problemas de coordinación es su potencial recurrencia, lo que invita a mantener de manera continua una ligera presión de demanda, previniendo el sesgo inflacionario con políticas dinámicas de oferta.

Como lo dice Dreze (2000): 'el debate en torno a la política económica me ha convencido que el principal obstáculo a la implementación de políticas eficaces viene de lagunas de la teoría macroeconómica, en particular de su desdeño relativo por la demanda agregada'.

Si se considera que las teorías de histéresis ${ }^{4}$ tienen alguna validez, un desafío importante para las autoridades es evitar una gestión excesivamente conservadora. En la medida en que no exista un relajo de la política económica cuando las condiciones así lo ameriten, el desempleo puede persistir, y, por la vía de la histéresis, validar estimaciones demasiado altas del desempleo estructural. El conservadurismo es de este modo autovalidante (Alsopp y Vines, 1998): se estima un dato de desempleo estructural alto (y un PIB potencial inferior), lo que lleva a una política económica

\footnotetext{
${ }^{4}$ En estricto rigor, la palabra histéresis debería utilizarse sólo cuando el equilibrio estacionario depende de su trayectoria (por ejemplo, si la tasa de desempleo efectiva afecta la tasa de desempleo de equilibrio). Con mayor frecuencia se utiliza el término para caracterizar situaciones en que las condiciones actuales afectan las condiciones de equilibrio por un tiempo prolongado.
} 
conservadora y a un desempleo persistente que se transforma en estructural.

En los modelos de histéresis, la tasa de desempleo natural está determinada por la política macroeconómica y por su propia historia. El desempleo cíclico, generado por un entorno recesivo prolongado, puede transformarse con el correr del tiempo en desempleo estructural. Por el lado de la oferta de trabajo, el desempleado de largo plazo se adapta a su situación, renunciando incluso a buscar un trabajo formal. Esto se traduce en una tasa natural de desempleo más alta, en la medida en que estos desempleados no presionan la evolución de los salarios (Blanchard, 1997). Por el lado de la demanda, el empresario privilegia la contratación de desempleados recientes más que de cesantes de mayor tiempo, simplemente porque su inserción puede ser más sencilla. Estos comportamientos también conducen a una tasa de desempleo natural más elevada. Con tasas de desempleo persistentemente altas, el concepto de tasa de desempleo neutral es un indicador engañoso para la política económica.

Como pone de relieve Stiglitz (1999), las consecuencias de las acciones no son sólo inciertas, sino también costosas de revertir. Será difícil recuperar un cliente cuando éste ha encontrado otro proveedor, y aun más difícil recontratar a un trabajador que ha encontrado otro trabajo. El supuesto de histéresis, entendido como la irreversibilidad producida por shocks negativos, tiene importantes consecuencias de política.

En la visión convencional del ciclo económico las fluctuaciones no representan otra cosa que desviaciones temporales en torno a una tendencia del producto. En los modelos de histéresis, en que cambios temporales (por ejemplo, movimientos de la demanda agregada en conjunto con ajustes lentos de precios) tienen efectos persistentes si un shock nominal en un marco de precios rígidos produce una caída en la demanda, el nivel de producto va a ser inferior al que se hubiese dado en ausencia de la perturbación, incluso después de que los precios se ajusten completamente. Los modelos que incorporan rigideces tienen así impactos muy diferentes en términos de bienestar, pues suponen una asimetría entre los episodios de expansión y de contracción de demanda. Los primeros aumentan el bienestar social y los segundos los disminuyen. La asimetría entre expansiones y recesiones es posible desde el momento en que la tasa natural del producto está por debajo de su nivel óptimo de manera persistente, dados los supuestos de competencia imperfecta.

Las caídas del producto respecto de su nivel de equilibrio tienen entonces grandes costos en términos de bienestar si afectan las decisiones de inversión y, por ende, el producto potencial. De ser correcto este punto de vista, las políticas de estabilización contracíclicas podrían significar ganancias significativas de bienestar (Romer, 1993). Así, la alta variabilidad de algunos precios, combinada con la rigidez relativa de otros, tiene un papel importante en la propagación y ampliación de las perturbaciones. Los riesgos asociados al ajuste de los precios pueden ser superiores a los vinculados a ajustes de cantidad, y por ello las cantidades pueden variar fuertemente. En un marco de incertidumbre y de rigideces de precios la volatilidad real es superior a la nominal.

En el planeta globalizado, las fuentes de incertidumbre se multiplican y las autoridades se ven enfrentadas a numerosos desafíos, pues deben conjugar la credibilidad de sus acciones con la flexibilidad necesaria para superar situaciones imprevistas. En esta encrucijada (la credibilidad supone reglas del juego estables durante un tiempo prolongado, la flexibilidad implica capacidad de respuesta ante cambios en las condiciones externas al sistema), muchos privilegian como único objetivo de la política económica la credibilidad de corto plazo, la que se lograría estableciendo metas presupuestarias rígidas, con la renuncia voluntaria a cualquier posibilidad de reacción frente a eventos adversos. Ante frecuentes perturbaciones asimétricas entre países, regiones y sectores, el criterio de disciplina no puede ser el único ni el dominante cuando las autoridades se enfrentan a múltiples situaciones que requieren de intervenciones discrecionales y transitorias por naturaleza, que son fundamentales para atenuar los fenómenos de persistencia. 


\section{III}

\section{Reglas macrofiscales para la inversión y el crecimiento}

En presencia de incertidumbre no hay lugar para reglas estrictas y concepciones rígidas, como tampoco para la improvisación y la incapacidad de reacción. Si los objetivos de la política fiscal son los de acometer de manera simultánea metas de crecimiento económico y de sostenibilidad de sus propias cuentas, es preciso adecuar las normas según criterios complementarios de disciplina fiscal y flexibilidad presupuestaria. La incertidumbre obliga a realizar frecuentes acciones discrecionales estabilizadoras y a evitar una gestión excesivamente conservadora.

De manera inédita, en la mayoría de los países de América Latina se han echado las bases de una gestión sana y eficiente de las finanzas públicas, con la promulgación reciente de leyes de responsabilidad fiscal. Pero quedan desafíos pendientes, especialmente en lo que se refiere al tratamiento del ciclo macroeconómico en la programación presupuestaria y al papel estabilizador de la política fiscal, aspectos fundamentales para lograr una adecuada complementariedad entre inversión pública y privada. Parece la ocasión para plantear estrategias de mediano plazo que dejen atrás los conflictos coyunturales. A pesar de los avances en materia de programación presupuestaria, y habida cuenta de las crónicas dificultades de financiamiento del sector público, las recientes reglas de política fiscal tienden aún a privilegiar metas de corto plazo, que no sobrepasan el ciclo presupuestario y que no incorporan cláusulas que permitan integrar las situaciones imprevistas que se producen de manera recurrente. El propio Manual de transparencia fiscal (FMI, 2001) advierte sobre el particular. Dicho Manual propone que cualquier regla adoptada por un gobierno debe estar claramente especificada. Obviamente, para que una regla fiscal sea duradera tiene que haber alguna flexibilidad en su aplicación cuando una desviación se justifica por las condiciones económicas.

En algunos casos, se establecen metas cuantificadas por ley, lo que elimina la posibilidad de que los estabilizadores automáticos operen plenamente $\mathrm{y}$, por lo tanto, no contempla los efectos del ciclo sobre el presupuesto. No puede existir sanción social o externa al desempeño presupuestario por cambios en varia- bles exógenas al sector público. Lo adecuado es incorporar en la propia programación cálculos conservadores o, en su defecto, contemplar mecanismos explícitos de desviación. No se puede calificar como un esquema de gestión eficiente a aquel, tan común en nuestra región, en que se producen ajustes continuos de gasto por desviaciones respecto a metas trimestrales de saldo difíciles de cumplir.

En algunos países se plantea en la ley que la tasa real de incremento del gasto público primario no podrá superar la tasa de aumento real del PIB. Dado el entorno incierto en que se desenvuelven las finanzas públicas, normas de este tipo parecen excesivamente rígidas. Por un lado, no se pondera adecuadamente el principio de estabilidad, al eliminar la capacidad de reacción de las autoridades ante situaciones recesivas (por ejemplo, con programas extraordinarios de empleos de emergencia). Por otro lado, se impone un techo poco realista de crecimiento del gasto, al establecerse sobre el PIB efectivo (conocido ex post) y no sobre el PIB potencial (definido ex ante). Parece más adecuado establecer criterios de crecimiento del gasto primario a partir del PIB potencial, eliminando así los efectos no deseados de las fluctuaciones cíclicas sobre la programación y la ejecución del gasto e introduciendo un significativo componente anticíclico.

Las normas de gasto no están sin embargo exentas de riesgos, por cuanto el tamaño del sector público depende de factores que no están directamente bajo el control de las autoridades, como las variables demográficas y económicas. Para una muestra de 125 países, Rodrik (1998) establece una relación positiva entre el tamaño del Estado -medido como la relación entre el consumo del gobierno y el PIB- y el grado de apertura al exterior. En palabras del autor: 'la asociación estadística entre la apertura y el gasto de Gobierno parece ser robusta. No es una relación espuria generada por variables omitidas. Ni es un artilugio de la muestra de países seleccionados o de una fuente de datos específica. La cuestión es por qué esta relación existe'. La explicación parece estar en que las economías más abiertas tienen una mayor exposición a las turbulencias de los mercados mundiales, y que estos 
riesgos se transmiten con mayor intensidad a las economías domésticas. El gobierno cumple una función de aislamiento de las turbulencias, siendo un sector "seguro" en comparación con el sector transable en el ámbito internacional. La vulnerabilidad externa puede empujar hacia un mayor peso del sector público en la economía en las etapas de transición, por lo que programar de manera automática el gasto primario sin considerar estos mecanismos puede ser difícil de lograr.

En general, las leyes de nuestros países son mucho más severas que las vigentes en los países desarrollados (Martner, 2000). Curiosamente, se busca ganar credibilidad macroeconómica por vías legislativas; pero la fe en las reglas o en las leyes no puede reemplazar el ejercicio de políticas responsables. La credibilidad de las políticas tiene más que ver con la capacidad de internalizar externalidades, es decir con cuerpos legales que integran las consecuencias de cambios en el entorno. Los objetivos principales de la política fiscal deberían definirse de la siguiente manera:

- En el mediano plazo, mantener finanzas públicas sanas: i) estableciendo prioridades de gasto y de tributación de manera de evitar alzas insostenibles de la deuda pública y/o tasas tributarias excesivas y ii) asegurando, en la medida de lo posible, que los costos de los servicios que se consumen sean pagados por la misma generación que se beneficia del gasto público;

- En el corto plazo, ayudar a la política monetaria: i) permitiendo el pleno funcionamiento de los estabilizadores automáticos en su papel de suavizamiento de las fluctuaciones macroeconómicas cuando existen variaciones de la demanda agregada, y ii) cuando sea prudente y apropiado, otorgando ayuda adicional mediante cambios en las políticas discrecionales.

Por cierto, los objetivos de corto y de mediano plazo están vinculados entre sí. Por ejemplo, la posibilidad de ayudar a la política monetaria a estabilizar la economía durante un episodio recesivo depende de la fortaleza de la posición financiera de mediano plazo del sector público.

Hay, sin embargo, un problema crítico con las instituciones fiscales. Cuando los acontecimientos aconsejan un giro en la orientación de la política fiscal, el cambio es muy difícil de lograr rápidamente, por la propia complejidad organizativa del sector público. Por ejemplo, las variaciones de impuestos requeridas por la coyuntura pueden llevar a intensas y largas negociaciones legislativas. Existen variadas propuestas para lograr una mayor autonomía en este ámbito. Una iniciativa interesante es la planteada en Australia. El Business Council of Australia (Gruen, 2001) ha propuesto que los instrumentos pertinentes debieran ser los impuestos al ingreso, tanto de las personas como de las sociedades. No sería necesario modificar las tasas legisladas por razones coyunturales si se introdujese un ponderador variable según la posición de la economía en el ciclo. El parámetro fiscal sería fijado inicialmente por el poder ejecutivo en un rango de 0.97 a 1.03, lo que significaría un amortiguador tributario no superior a un punto del PIB.

Por el lado de los gastos, los cambios necesitan por lo general de autorización presupuestaria; además, el diseño y evaluación de los proyectos toma tiempo. De hecho, en la historia de los Estados Unidos muchos de los estímulos discrecionales se han aplicado cuando la recesión ya estaba técnicamente terminada (Gruen, 2001). Otro problema muy serio es el riesgo de irreversibilidad asociado a los impulsos fiscales, en principio transitorios. La tendencia al sesgo de déficit, generado por la dificultad de revertir los procesos, es uno de los principales argumentos para impulsar el establecimiento de reglas obligatorias que limiten este tipo de acciones discrecionales.

A pesar de los riesgos, cabe destacar la posición de Ball (1996) respecto de la combinación óptima de políticas: 'Los rezagos más cortos (en su impacto sobre el gasto) son la principal ventaja para usar la política fiscal (más que la política monetaria) como herramienta macroeconómica. Si los hacedores de políticas usaran sus instrumentos fiscales cometerían menos errores. Y los errores podrían corregirse más rápidamente'.

La siguiente ecuación del déficit público ilustra bien la problemática de la política fiscal:

$$
d=d_{e}-(\alpha+\beta) G A P
$$

donde $d$ es el déficit público efectivo, $d_{e}$ representa el componente estructural del déficit público, $\alpha$ la sensibilidad marginal del déficit a la brecha de PІв (o déficit cíclico), $\beta$ la reacción discrecional de las autoridades al ciclo (o déficit discrecional) y GAP la brecha de PIB. Cualquier regla macrofiscal debe tener en cuenta estos tres elementos: un objetivo de déficit estructural de mediano plazo, cláusulas de excepción y de transitoriedad cuando existan fluctuaciones macroeconómicas imprevistas, y algún margen de maniobra para afrontar situaciones recesivas persistentes (Buti, Franco y Ongena, 1998). Es lo que se hace en las legislaciones más recientes de algunos países. 
Por ejemplo, en Nueva Zelandia la Ley de responsabilidad fiscal (Fiscal Responsibility Act) de 1994 estableció como criterios 'mantener a niveles prudentes la deuda pública asegurando, en promedio y a lo largo de un período razonable, que los gastos corrientes no excedan los ingresos corrientes'. ${ }^{5}$ La definición de un nivel "prudente" de deuda que permita un cierto margen de maniobra en la eventualidad de eventos futuros adversos no se especifica en la legislación. No existe un nivel de deuda que pueda ser considerado prudente para todos los tiempos. Todos los factores relevantes, como la vulnerabilidad ante shocks externos, el costo del servicio de la deuda, las presiones demográficas y otros, cambiarán probablemente a lo largo del tiempo.

Los gobiernos pueden apartarse temporalmente del principio de prudencia, pero la legislación estipula que se deben precisar las razones de esta desviación, y cómo se prevé volver a tal nivel. El propósito es evitar los problemas asociados con los objetivos numéricos en la legislación; la dificultad de anticipar el futuro lleva a la necesidad de alguna flexibilidad de corto plazo, pero con desvíos temporales y transparentes.

Las leyes del Reino Unido se guían por una filosofía similar. El Código de estabilidad fiscal, aprobado por la Cámara de los Comunes en diciembre de 1998, establece los criterios que deben guiar la formulación e implementación de la política fiscal. Las reglas fiscales gubernamentales inicialmente fueron fijadas en julio de 1997 en el Informe financiero y presupuestario y fueron confirmadas en el presupuesto de marzo de 1998. Nótese que no se incorporan en el código de estabilidad fiscal, sino en las leyes anuales de presupuesto. ${ }^{6}$ Una aproximación alternativa sería incluir estas reglas fiscales en la Ley, pero ello sería excesivamente restrictivo, puesto que el proceso en marcha podría requerir que las reglas fuesen complementadas. Más aun, según el criterio imperante, le corresponde a cada gobierno elegido escoger y anunciar los objetivos y reglas de sus políticas, siempre que sean consistentes con los principios fiscales establecidos en la Ley. El gobierno establece dos reglas para la legislatura: i) la regla de oro: a lo largo del ciclo, el gobierno se endeuda sólo para invertir y no para cubrir gastos corrientes; ii) la regla de inversión sostenible: la deuda pública se mantendrá, como proporción del ingreso nacional, a un nivel estable y prudente a lo largo del ciclo.

La regla de oro promueve la ecuanimidad entre generaciones, al asegurar que la cuenta de los gastos

\footnotetext{
${ }^{5}$ Véase Nueva Zelandia, The Treasury (1995).

${ }^{6}$ Véase Reino Unido, Her Majesty's Treasury (1998 y 1999).
}

de hoy, que benefician principalmente a los contribuyentes actuales, no se traspasará a las generaciones futuras. Por el contrario, la inversión de hoy beneficiará tanto a las futuras generaciones como a las actuales. Ello no implica que el gasto en capital tenga un rango automáticamente superior al gasto corriente; ambos juegan un papel, y ambos tienen efectos duraderos en la economía. La regla de oro se aplica a la inversión neta; se implementa usando una definición de la cuenta corriente del sector público cercana al concepto de Cuentas Nacionales, de manera que la depreciación del capital público se incluye como gasto corriente. Se asegura así que los contribuyentes actuales pagan el costo de mantener el stock de capital. La definición de Cuentas Nacionales es transparente y evita la tentación de hacer pasar gastos corrientes por gastos de capital para cumplir la regla. En cuanto a la segunda regla, el Gobierno estipula que, todo lo demás igual, es deseable reducir por debajo del $40 \%$ la deuda pública neta a lo largo del ciclo. Un objetivo de deuda definido a lo largo del ciclo permite tomar en cuenta el entorno macroeconómico, al cual este indicador es muy sensible, especialmente en cuanto al diferencial entre tasa de crecimiento y tasa de interés sobre la deuda.

Otra normativa legal de gran importancia es la del Pacto por la Estabilidad y el Crecimiento de la Unión Europea, la que establece objetivos de mediano plazo respecto de posiciones fiscales en equilibrio o en superávit, y compromete a sus miembros a presentar programas de estabilidad trianuales que especifiquen las trayectorias contempladas para acometer tales objetivos. La resolución de Amsterdam supone que la "adhesión al objetivo de una posición presupuestaria cercana al equilibrio o en excedente permitirá a todos los Estados miembros enfrentar las fluctuaciones cíclicas normales, manteniendo el déficit dentro del valor de referencia de 3\% del PIB". El intervalo de tiempo que permite interpretar el mediano plazo es el ciclo macroeconómico.

Para juzgar el grado de cumplimiento de los objetivos de mediano plazo, en la práctica se debe evaluar la incidencia probable de la coyuntura sobre la evolución actual y futura de las cuentas públicas, con algún método aceptado por todos los Estados miembros. Tanto los Estados miembros como el Comité del Banco Central Europeo ${ }^{7}$ consideran el método de los

\footnotetext{
7 Véase en Consejo de la Unión Europea (1998) la opinión del Comité Monetario sobre el contenido y formato de los programas de estabilidad y convergencia.
} 
servicios de la Comisión como apropiado y útil para examinar para cada Estado miembro los saldos públicos cíclicamente ajustados.

Todos los países someten, a partir de 1999 y a principios de cada año, al Consejo y a la Comisión programas trianuales de estabilidad (para los países de la zona del euro) o de convergencia (para los restantes) que responden al criterio del Pacto. Según el primer reglamento del Consejo, ${ }^{8}$ que tiene vigencia legal desde el 1 de julio de 1998, los programas de estabilidad y convergencia deberán presentar la siguiente información:

i) El objetivo de mediano plazo de una posición presupuestaria cercana al equilibrio o en excedente y la trayectoria de ajuste hacia este objetivo para el saldo del Gobierno General, y la trayectoria esperada para la relación deuda sobre PIB del Gobierno General;

ii) Los principales supuestos relativos a los acontecimientos esperados y a las variables económicas que son relevantes para la realización de los programas de estabilización, como el gasto en inversión de Gobierno, el crecimiento del PIB, el empleo y la inflación;

iii) Una descripción de las medidas presupuestarias $u$ otras que se proponen para lograr los objetivos del programa y, en el caso de las medidas presupuestarias principales, una estimación de sus efectos cuantitativos sobre el presupuesto.

iv) Un análisis de cómo los cambios en los supuestos económicos principales tienen incidencia en el saldo y en la deuda pública.

La información referida a las trayectorias del saldo público, de la deuda y de las principales variables económicas reseñadas se entregará sobre una base anual y deberá cubrir el año anterior, el año en curso y los tres siguientes años. Los Estados miembros deberán hacer públicos sus programas de estabilidad y convergencia anualmente. El Comité ha elaborado un informe técnico, entendido como un código de buenas prácticas, relativo al formato y al contenido de los programas de estabilidad y convergencia, de manera a facilitar su examen y discusión. Los principales elementos de estos programas son los siguientes:

i) Los supuestos sobre el crecimiento del PIB y de las fuentes esperadas de este crecimiento deben ser fundamentados, proveyéndose suficiente información

\footnotetext{
8 Véase en Consejo de la Unión Europea (1997a) la Council Regulation (EC) sobre el fortalecimiento de la vigilancia de las posiciones presupuestarias y la vigilancia y coordinación de las políticas económicas.
}

para analizar la posición cíclica de la economía. También deben presentarse los supuestos técnicos sobre la evolución de las tasas de interés, dado su impacto sobre las finanzas públicas. Ante las dificultades prácticas para el uso de un conjunto común de proyecciones macroeconómicas, el Comité prefirió que los países miembros establezcan sus propias previsiones sobre la economía doméstica y la situación mundial. Sin embargo, si existen diferencias significativas con las proyecciones de la Comisión, el país miembro debe justificar sus presunciones.

ii) Los programas deben incluir análisis de sensibilidad, estimando cómo afectaría los cambios en los principales supuestos económicos al saldo y a la deuda pública. Este análisis debe ser complementado por un estudio sobre el impacto de diferentes supuestos de tasa de interés sobre el déficit y la deuda.

iii) La información sobre las trayectorias del déficit y de la deuda del Gobierno General y de los supuestos sobre las principales variables económicas debe cubrir al menos los tres próximos años, dejando abierta la posibilidad de presentar un período más largo si algún país miembro así lo desea.

iv) Las actualizaciones anuales debieran mostrar cómo se han comportado las variables respecto de los objetivos del programa anterior y, cuando ocurren desviaciones significativas, incluir las etapas consideradas para rectificar la situación.

El Pacto permite, sin embargo, interpretar el objetivo de saldo de manera más flexible, aceptándose déficit superiores, aunque acotados, originados en factores cíclicos transitorios. Los procedimientos están estipulados en el Protocol on Excesive Deficit Procedure, ${ }^{9}$ artículo 2:

i) El exceso de déficit de gobierno por sobre el valor de referencia será considerado excepcional cuando resulte de un evento inusual fuera del control del gobierno miembro y que tiene un impacto significativo sobre la posición financiera del gobierno general, o cuando resulte de un severo cambio coyuntural desfavorable. Además, el exceso será considerado transitorio si los servicios de previsión de la Comisión indican que el déficit volverá a situarse por debajo del valor de referencia después de que termine el evento inusual o el cambio coyuntural desfavorable. La corrección del

\footnotetext{
9 Véase en Consejo de la Unión Europea (1997b) la Council Regulation (EC) $\mathrm{N}^{\circ} 1467 / 97$ respecto a acelerar y aclarar la aplicación del procedimiento de déficit excesivo, vigente a partir del $1 \mathrm{de}$ enero de 1999.
} 
déficit excesivo debería completarse dos años después de su ocurrencia, y un año después de su identificación, a menos que existan circunstancias excepcionales. Las estadísticas utilizadas para la aplicación del protocolo serán provistas por la Comisión.

ii) La Comisión considerará, como una regla, que el exceso de déficit será excepcional sólo si existe una caída interanual del PIB de por lo menos $2 \%$.

iii) La Comisión decidirá si la situación es excepcional en el caso de caídas menores al $2 \%$ y existe un exceso de déficit, tomando en cuenta las observaciones realizadas por el país miembro, en particular relativas a lo abrupto de la recesión y a la acumulación de pérdidas de PIB respecto de las tendencias pasadas.

Se trata de combinar disciplina con flexibilidad, con una programación plurianual con objetivos explí- citos y una gestión de corto plazo más atenta a las fluctuaciones macroeconómicas. Estos elementos ayudan a mejorar la eficiencia y eficacia de las políticas públicas, al alargar el horizonte de la gestión pública, y reservar márgenes de maniobra para enfrentar situaciones imprevistas. Esto es posible sólo en la medida en que exista transparencia y mecanismos claros de rendición de cuentas. La combinación de metas de mediano plazo debidamente informadas (una situación cercana al equilibrio o en superávit en condiciones normales en la zona euro, equilibrio en cuenta corriente en el ciclo y deuda pública estable en el ciclo en el Reino Unido) y de una programación presupuestaria en que se define la trayectoria para acometer esos objetivos permite apreciar la situación de las finanzas públicas y su orientación.

\section{IV}

\section{Una gestión pública para el futuro}

A medida que la estabilidad macroeconómica permite visualizar un horizonte de mediano plazo, se hace cada vez más posible y necesario el rescate de la planificación, como un instrumento fundamental de la gestión pública en América Latina. Este proceso se ha ido profundizando de una manera gradual y no en la forma de un proyecto previamente concebido. La planificación debe ilustrar las perspectivas a mediano y largo plazo para el conjunto de los ciudadanos, clarificar las alternativas de decisión de las autoridades públicas, explorar las nuevas estrategias económicas y sociales posibles y deseables. La planificación construye un puente entre los grandes objetivos políticos y económicos del Gobierno y la ejecución de sus planes y programas.

Realizar un esfuerzo de prospectiva para incorporar anticipación y un horizonte de largo plazo en el proceso de toma de decisiones es una de las tareas decisivas de la gestión pública. Como herencia de los ajustes realizados desde los años ochenta, el grueso de los países de América Latina tiene horizontes temporales muy limitados. Pese a ello, es misión indelegable del Estado preparar el futuro, mejorar la capacidad de anticipación, resistir a la "tiranía destructiva del corto plazo", ofrecer visiones de largo alcance. La anticipación es una condición de la acción, y ésta no puede confundirse con una mera gestión de las urgencias. El porvenir no sólo se prevé, también se construye: an- ticipar para actuar a favor de un futuro deseable libremente consensuado, tal es el objeto de la prospectiva.

Podemos distinguir dos visiones polares de la prospectiva: una de naturaleza exploratoria, que parte del presente para recorrer el espectro de los futuros posibles, y otra normativa, que parte de una visión del futuro deseable y construye el itinerario de las acciones necesarias para realizarlo. Si se parte de la realidad, el riesgo es quedarse en ella, sin cambiar nada, o sólo en el margen. Si se parte del imaginario, el riesgo también es quedarse en él, construyendo sueños. Lo natural es buscar imágenes del futuro a partir del presente, pero el itinerario inverso, partir del imaginario, es atractivo, pues lo esencial es romper las inercias y movilizar energías. El desafío, en este último caso, es transformar estas ideas en nuevas estrategias económicas y sociales que permitan enfrentar los grandes problemas de la región.

Preparar la gestión pública para el futuro supone avanzar en siete áreas bien definidas (OCDE, 2001a): i) reglas macrofiscales, ii) presupuestación plurianual, iii) presupuesto base cero, iv) relajación de controles internos, v) contabilidad y gestión sobre base devengado, vi) evaluación de resultados y vii) convenios de desempeño. Nótese que esta clasificación supone una secuencia. Así, la evaluación de resultados sólo tiene sentido si se implementan las reformas anteriores. 
Como es sabido, la gestión pública ha evolucionado del presupuesto por programas al enfoque por resultados. La técnica de presupuesto por programas procuraba establecer vínculos estrechos entre el proceso presupuestario, la planificación y la evaluación de los programas públicos. En su concepción más amplia, el enfoque por resultados busca más bien enriquecer la discusión presupuestaria en un marco flexible (véase Marcel, 1998). El proceso de modernización de la gestión de las instituciones está muy vinculado en la actualidad al modelo denominado modelo de gestión por resultados, lo que supone avanzar simultáneamente en una cierta autonomía en la toma de decisiones de los centros gestores y en la construcción de sistemas apropiados de evaluación. Esta última debe ser a la vez interna, a través de indicadores y de metas de desempeño, y externa, a partir de rondas evaluatorias realizadas por otros organismos. Los criterios orientadores tienen que ver con la planificación estratégica de los organismos públicos, el tipo de vinculación entre la asignación de recursos y el desempeño institucional, la transparencia del accionar del Estado, y, como corolario de lo anterior, la búsqueda del cambio de cultura organizacional de las entidades públicas.

Este proceso no está exento de dificultades, pues debe enfrentar obstáculos en las propias deficiencias actuales (pasar de una cultura de procedimientos a una de resultados, orientada al usuario) y en las limitaciones externas (continuos cambios refundacionales que impiden la emergencia de una estructura orgánica adecuada, con recursos humanos especializados). Cada experiencia es única; los factores específicos a cada país e institución hacen que instrumentos y procedimientos difieran en cada caso.

Pero es claro que la gestión por resultados sólo pasará a ser una realidad cotidiana si se refuerza la capacidad de autonomía de las agencias, a través de convenios de desempeño. Estos convenios tienen varios propósitos: aumentar la eficiencia/eficacia, la responsabilidad (accountability) y la capacidad gerencial, pasar de un enfoque de insumos y reglas a otro de productos y resultados, construir confianza. En general, los contratos de desempeño no son legales, sino mutuamente negociados sobre la base de acuerdos entre ministros y jefes ejecutivos, o entre direcciones y agencias. Estos contratos se generan a partir de acuerdos, los que sirven de base para resolver disputas, enfrentar contingencias o ajustes cuando existen eventos no previstos. Los contratos de desempeño se construyen sobre la base de un modelo contractual "relacional" y no clásico, y sacan su fuerza no por la ame- naza de sanciones legales o financieras, sino de la necesidad de las partes de tener relaciones claras y acuerdos estables (OCDE, 1999).

La transformación de la administración pública puede generar una incompatibilidad entre la necesidad de control central de las operaciones y la libertad gerencial requerida para mejorar el desempeño. En este tema es muy importante no confundir, por el lado de las oficinas de presupuestos, los nuevos sistemas de gestión pública con los ajustes fiscales de corto plazo, y por el lado de los gerentes, las reformas con una licencia para gastar como quieran (Shick, 2001). La innovación gerencial supone nuevos modelos de relacionamiento entre "gastadores" y "asignadores", para buscar un adecuado equilibrio entre la necesidad de flexibilidad en la implementación de planes y programas y la disciplina que supone formar parte de un sector público con reglas macrofiscales explícitas.

Resulta indispensable clarificar las decisiones políticas con una visión plurianual. La realización de planes y programas públicos debe insertarse en un marco de presupuestación plurianual, y ello no es otra cosa que planificar con sentido estratégico. Hasta hace algunos años la plurianualidad era sinónimo de rigidez presupuestaria, entendida como la acumulación de compromisos sectoriales radicalmente incompatibles con los objetivos globales. Esta "mala" plurianualidad ha dado paso a una visión más optimista de programación de las finanzas públicas. Hoy en día, el ejercicio de una "buena" plurianualidad es la consecuencia natural del auge de los convenios de desempeño y de los instrumentos de evaluación de las políticas públicas. No es nuevo utilizar la perspectiva multianual para la gestión pública; la innovación consiste en lograr una articulación creciente entre plan, presupuesto y evaluación de resultados, formalizar procesos en torno a estos instrumentos de manera de asegurar consistenciatemporal en la toma de decisiones, diseñar un eslabonamiento que le otorgue viabilidad al modelo de gestión por resultados.

La gestión pública podría facilitar el proceso de toma de decisiones y los arbitrajes, tanto a nivel central como regional, si es diseñada a partir de ejercicios prospectivos y de planes estratégicos, con reglas fiscales de mediano plazo, programación presupuestaria plurianual, convenios de desempeño, instancias de coordinación efectiva y sistemas abiertos de evaluación de planes y programas. Si la planificación ha de desempeñar adecuadamente sus funciones, se hace necesario introducir más pragmatismo y establecer las bases de una gestión flexible, descentralizada y con 
mayor responsabilidad y capacidad de acción de sus actores.

Para hacer consistentes las reglas de mediano plazo con la gestión del gasto público, el nuevo régimen de planificación y de control debe evitar, por una parte, la tendencia al cortoplacismo en las decisiones, y el incrementalismo en el manejo del presupuesto, y por otra, el sesgo negativo que generalmente afecta al gasto en inversión. La clara separación de los gastos corrientes, incluyendo la depreciación, y de capital, y la asignación de proporciones crecientes del gasto público de manera plurianual son aspectos que sin duda pueden ser decisivos en la ardua tarea de construir un entorno institucional favorable a la estabilidad y el crecimiento.

Asegurar un entorno apropiado para la inversión privada y administrar adecuadamente los escasos recursos disponibles para la inversión pública, supone una gestión capaz de enfrentar tres desafíos fundamentales: el primero, respetar una regla fiscal a lo largo del ciclo, para evitar los costos económicos y políticos de los ajustes fiscales abruptos; el segundo, identificar con la debida anticipación los déficit estructurales, de manera a evitar un endeudamiento público excesivo que represente una carga para las futuras generaciones; y el tercero, eliminar el sesgo en contra de los gastos de capital. Estos son en general más sensibles a los ajustes fiscales que los gastos corrientes, por su propia naturaleza. Postergarlos o no realizarlos, también constituye una carga para las generaciones futuras.

En lo que se refiere al primer desafío — respetar la regla fiscal a lo largo del ciclo-, se trata de desarrollar instrumentos que orienten el proceso presupuestario hacia un esquema de disciplina y flexibilidad, en que se identifiquen con claridad los factores transitorios y se asegure una conducción consistente con el ineludible pacto fiscal que requieren nuestras sociedades. El criterio relevante parece ser buscar una posición financiera corregida por las fluctuaciones del nivel de actividad, lo que equivale a programar los gastos e ingresos con una visión de mediano plazo en el manejo de las finanzas públicas. Cuando se diseñan las políticas presupuestarias, se debe tomar en cuenta que la marcha cíclica de la economía es inevitablemente incierta y que las proyecciones de los determinantes de ingresos y gastos son necesariamente imprecisas.

Para enfrentar adecuadamente el segundo desafío -identificar con la debida anticipación los déficit estructurales-, es necesario asegurar una trayectoria plurianual consistente con la regla fiscal. La mayor parte de los errores en la programación plurianual es atribui- ble a los desaciertos de previsión sobre el potencial de crecimiento de las economías, y estos errores tienen efectos permanentes en las finanzas públicas. Si el PIB efectivo es inferior al tendencial estimado durante el lapso considerado en los planes de Gobierno, el resultado es una merma estructural de la posición financiera del sector público.

Es importante entonces tomar en cuenta explícitamente la posición de la economía en el ciclo y adoptar supuestos moderados de crecimiento en la programación plurianual. De manera deliberada, y aprendiendo de las lecciones del pasado, como la observación de que la principal causa de fracaso de las experiencias de programación plurianual del presupuesto es el excesivo optimismo respecto del crecimiento de mediano plazo, se debe emprender una estrategia prudente, que permita ajustarse a las fluctuaciones macroeconómicas.

Parece necesario encarar este "sesgo de optimismo" (en que los episodios positivos son considerados como permanentes y los negativos como transitorios) para asegurar una programación fiscal más consistente y más transparente. Los análisis de sensibilidad no deben limitarse a construir escenarios con distintos valores de las previsiones del PIB a un año, sino también contemplar trayectorias menos optimistas respecto del PIB tendencial. Se dispondría así de una trayectoria plurianual prudente, requerida en un entorno incierto para asegurar márgenes de seguridad e internalizar de esta manera la eventualidad de imprevistos y de errores de medición en la propia programación presupuestaria.

Orientar la política fiscal en función de un objetivo estructural de mediano plazo va mucho más allá de un simple criterio, pues supone efectuar mediciones sistemáticas de la posición de la economía en el ciclo, y por ende de los elementos que están afectando el PIB potencial. La gestión pública debiera tener un fuerte componente de análisis macroeconómico, mucho más intenso de lo habitual en nuestros países.

En cuanto al tercer desafío - eliminar el sesgo en contra de los gastos de capital-, es importante reconocer explícitamente en la programación presupuestaria la diferencia económica entre el gasto corriente y el de capital. El Estado tiene como deber alcanzar o mantener el stock de capital necesario para la economía y asegurar que su componente público se conserva en buenas condiciones. Un nivel inadecuado de inversión pública puede dañar irremediablemente el desempeño de largo plazo de las economías.

En muchos países se están realizando grandes esfuerzos para adecuar sus propios procesos presupues- 
tarios al objetivo de estimular y proteger el gasto en inversión pública. Es útil para ello planificar y gestionar separadamente los gastos corrientes y de capital. En el modelo de gestión por resultados, planificar, gestionar y contabilizar los gastos debe hacerse sobre una base de devengado, registrando en el momento en que ocurren los costos del capital, como la depreciación e intereses de la inversión pública y de los restantes activos. De esta manera, se asegura un mayor vínculo entre el proceso de planificación del gasto y la regla fiscal.

La experiencia reciente del Reino Unido ${ }^{10}$ parece particularmente interesante, pues se establecen planes "a firme" de tres años para todas las reparticiones de Gobierno, a través de los Departmental Expenditure Limits (DEL). Se espera que estos límites (más o menos la mitad del gasto total) representen una base sólida para la planificación y un fuerte incentivo para administrar los costos de manera eficiente. El Gobierno busca también una mayor flexibilidad de gestión para mejorarla, aceptando que las agencias tengan la libertad de trasladar cualquier parte de sus gastos incluidos en los DEL de un año a otro.

Cuando el gasto no puede, razonablemente, ser objeto de un plan trianual, es sujeto de un escrutinio anual como parte del proceso presupuestario, y se le denomina gasto administrado anualmente, Annually Managed Expenditure (AME). La mayor parte de estos gastos tienen que ver con la Seguridad Social y están sujetos a un riguroso control anual. De manera consistente con la regla fiscal, los gastos corrientes y de inversión son planificados y gestionados separadamente, tanto dentro de los DEL como de los AME.

A partir del 2000, se implementa un nuevo sistema contable para el sector público sobre una base devengado, el que complementará las cuentas actuales base caja. El uso de principios de la contabilidad base devengado reconoce que los efectos económicos de los gastos de capital no son los mismos que los de gastos corrientes, y además registra los gastos en el momento en que se han incurrido y no cuando se han pagado. El Resource Accounting and Budgeting (RAB) se propone planificar, gestionar y contabilizar los DEL sobre una base de devengado, registrando en el momento en que ocurren los costos del capital, como la depreciación e intereses de la inversión pública y de los restantes activos.

${ }^{10}$ Véase al respecto Reino Unido, Her Majesty’s Treasury (2000).
De esta manera, se asegura un mayor vínculo entre el proceso de planificación del gasto y la regla fiscal, contabilizándose los gastos de las agencias sobre la misma base que se utiliza para realizar las proyecciones fiscales. Vale la pena notar, sin embargo, que el sistema de caja seguirá siendo relevante, por ejemplo, para contabilizar las necesidades financieras del Gobierno. Más aun, la previsión de impuestos continuará basándose en una base caja.

Se ha implementado plenamente una contabilidad con base devengada en Australia, Canadá, Nueva Zelandia, Islandia e Italia; otros países están desarrollando estos sistemas en la actualidad (ver OCDE, 2001b). La alta calidad de la información es la base para una buena política de toma de decisiones.

La gestión pública debe así combinar un diseño transparente, con reglas que aseguran el control de las finanzas públicas a mediano plazo, y un nuevo régimen de planificación presupuestaria, basado en la asignación plurianual de proporciones crecientes del gasto público. Estos dos pilares son inseparables; el primero (las reglas fiscales) le otorga factibilidad técnica al segundo (la programación plurianual), al establecer normas independientes del ciclo macroeconómico, y el segundo, otorga mejores incentivos a las agencias para manejar de manera más eficiente sus presupuestos y contribuir así al cumplimiento de las metas a lo largo del ciclo.

La estrategia debe entonces enfatizar la planificación de largo plazo, poner el acento en los productos más que en los insumos; distinguir con claridad los gastos corrientes y de capital, y finalmente basarse en la prudencia y la estabilidad, creando un margen para enfrentar las inevitables incertidumbres. Los desarrollos expuestos dan cuenta de interesantes cambios en la manera de encarar la gestión pública. Luego de casi dos décadas de declinación, la planificación, guiada por planes plurianuales y programas, permite revertir la administración por sectores e instituciones. El enfoque normativo da paso a la gestión estratégica y a la prospectiva. Ello supone incorporar la dimensión plurianual en planes de inversión y marcos presupuestarios; el desafío es coordinar las inversiones públicas y privadas para el crecimiento.

En un entorno dominado por desequilibrios y conflictos emergentes y variados, se trata de construir nuevos andamiajes institucionales, con esquemas prudenciales orientados a internalizar lo más posible externalidades positivas y negativas a través de reglas, procedimientos y protocolos de excepción. No se trata 
de decretar credibilidad por ley, sino de desarrollar estrategias de largo aliento comprometidas con la responsabilidad, la estabilidad y el crecimiento. El asunto no es meramente semántico: nuestros países requie- ren leyes de responsabilidad fiscal que ponderen adecuadamente el principio de responsabilidad, pero también es necesario pensar en leyes de estabilidad y crecimiento. ¡La diferencia no es menor!
Alsopp, C. y D. Vines (1998): The assessment: Macroeconomic policy after EMU, Oxford Review of Economic Policy, vol. 14, $\mathrm{N}^{\circ} 3$, Londres, Oxford University Press.

Ball, Lawrence (1996): A Proposal for the Next Macroeconomic Reform, Wellington, Victoria University of Wellington Foundation.

Blanchard, O. J. (1996): Macroeconomics, Upper Saddle River, New Jersey, Prentice Hall.

(1997): Comment on Ball: disinflation and the nairu, en C. Romer y D. Romer (eds.), Reducing Inflation. Motivations and Strategy, Chicago, Illinois, Chicago University Press.

Blanchard, O. J. y N. Kiyotaki (1987): Monopolistic competition and the effects of aggregate demand, The American Economic Review, vol. 77, № 4, Washington, D.C., American Economic Association.

Buti, M., D. Franco y H. Ongena (1998): Fiscal discipline and flexibility in EMU: The implementation of the stability and growth pact, Oxford Review of Economic Policy, vol. 14, $\mathrm{N}^{\circ} 3$, Londres, Oxford University Press.

Consejo de la Unión Europea (1997a): Council Regulation, № 1466/97, Bruselas, julio.

(1997b): Council Regulation, № 1467/97, Bruselas, julio. (1998): Opinion of the Monetary Committee on the Content and Format of Stability and Convergence Programmes, Bruselas, octubre.

Dreze, J.H. (1997): Walras-Keynes equilibria, coordinations and macroeconomics, European Economic Review, vol. 4 (9), Amsterdam, Países Bajos, Elsevier Science Publishers B.V. (2000): Sur la macroéconomie de l'incertitude et des marchés incomplets, Revue de l'OFCE, № 72 (7-37), París, Observatoire Français des Conjonctures Economiques.

FMI (Fondo Monetario Internacional) (2001): Revised Manual on Fiscal Transparency, Washington, FMI.

Friedman, M. (1968): The Role of Monetary Policy, The American Economic Review, vol. 58, № 1, Washington, D.C., American Economic Association.

Greenspan, A. (1996): The challenge of central banking in a democratic society, remarks at the Annual Dinner and Francis Boyer Lecture of the American Enterprise Institute for Public Policy Research, Washington D.C., The Federal Reserve Board.

Greenwald, B. y J. Stiglitz (1989): Toward a theory of rigidities, The American Economic Review, vol. 79, № 2, Washington, D.C., American Economic Association.

(1993): New and old keynesians, The Journal of Economic Perspectives, vol. 7, $\mathrm{N}^{\circ} 1$, Nashville, Tennessee, American Economic Association.

Gruen, N. (2001): Greater independence for fiscal institutions, $O E C D$ Journal on Budgeting, vol. 1, $\mathrm{N}^{\circ}$ 1, París, Organización de Cooperación y Desarrollo Económicos (OCDE).
Keynes, J. M. (1936): The General Theory of Employment, Interest and Money, Londres, MacMillan.

Mankiw, N. G. (1985): Small menu costs and large business cycles: A macroeconomic model of monopoly, The Quarterly Journal of Economics, vol. 100, № 2, Cambridge, Massachusetts, MIT Press. (1989): Real business cycles: A new Keynesian perspective, The Journal of Economic Perspectives, vol. 3, $\mathrm{N}^{\circ}$ 3, Nashville, Tennessee, American Economic Association.

(1993): Symposium on Keynesian economics today, The Journal of Economic Perspectives, vol. 7, $\mathrm{N}^{\circ} 1$, Nashville, Tennessee, American Economic Association.

Marcel, M. (1998): Indicadores de desempeño como instrumentos de la modernización del Estado en Chile, Perspectivas en política, economía y gestión, vol. 1, № 2, Santiago de Chile, Universidad de Chile, Facultad de Ciencias Físicas y Matemáticas, Departamento de Ingeniería Industrial.

Martner, R. (2000): Gestión pública y programación plurianual. Desafíos y experiencias recientes, serie Gestión pública, Santiago de Chile, CEPAL.

Nueva Zelandia, The Treasury (1995): The Fiscal Responsibility Act: An Explanation, Wellington.

OCDE (Organización de Cooperación y Desarrollo Económicos) (1999): Performance contracting lessons from performance contracting case studies, Public Management Service, París, OCDE.

(2001a): Government of the future, París, OCDE.

(2001b): Accrual accounting and budgeting practices in member countries overview (International Accounting and Budget Symposium, París, 13 y 14 de noviembre de 2000), París, OCDE.

Reino Unido, Her Majesty's Treasury (1998): The Code for Fiscal Stability, Londres.

(1999): Analysing UK Fiscal Policy, Londres.

(2000): Resource Budgeting and the 2000 Spending Review, Londres, julio.

Rodrik, D. (1998): Why do more open economies have bigger governments?, Journal of Political Economy, vol. 106, $\mathrm{N}^{\circ} 5$, Chicago, Illinois, The University of Chicago.

Romer, D. (1993): The new Keynesian synthesis, The Journal of Economic Perspectives, vol. 7, № 1, Nashville, Tennessee, American Economic Association.

Shick, A. (2001): The changing role of the central budget office, OECD Journal of Budgeting, vol. $\mathrm{N}^{\circ} 1$, París, OCDE, enero.

Stiglitz, J. (1999): Toward a general theory of wage and price rigidities and economic fluctuations, The American Economic Review, vol. 89, № 2, Washington, D.C., American Economic Association.

Tobin, J. (1993): Price flexibility and output stability: An old Keynesian view, The Journal of Economic Perspectives, vol. 7, № 1, Nashville, Tennessee, American Economic Association. 